\title{
电子信息工程技术的应用及未来发展研究
}

李 云

河南安金电子有限公司，河南 南阳 473000

[摘要]电子信息工程技术是一种复合型技术，其充分的将电子信息技术与通信技术进行有效的结合，属于超高创新型技术， 具有非常强的实用性与便捷性，给人们的生活、工作带来了巨大的改变。在不断使用的过程中，其在与其他学科的技术进行 结合后形成了全新的技术, 并得到了有效的应用, 主要包括控制技术, 进一步促进了电子技术的发展, 得到了交通行业、医 疗行业、农业领域及军事领域的充分认可与利用, 因此可以看出电子信息工程技术的应用前景非常广阔, 并给社会进步带来 巨大的影响。要想确保电子信息工程技术得到进一步的发展, 应对其进行更加深入的研究, 充分的了解其作用, 使其可以为 我们提供更好的服务。

[关键词]电子信息工程技术; 应用; 未来发展

DOI：10.33142/ec.v2i7.488 中图分类号：TN0 文献标识码：A

\section{Research on the Application and Future Development of Electronic Information Engineering Technology}

LI Yun

Henan Anxin Electronics Co., Ltd., Henan Nanyang, 473000 China

\begin{abstract}
Electronic information engineering technology is a kind of compound technology, which fully combines electronic information technology with communication technology, belongs to ultra-high innovative technology, has strong practicability and convenience, and has brought great changes to people's life and work. In the process of continuous use, it has formed a new technology after combining with the technology of other disciplines, and has been effectively applied, including control technology, which has further promoted the development of electronic technology, and has been fully recognized and utilized in the transportation industry, medical industry, agricultural field and military field. Therefore, we can see that electronic information engineering technology can be seen. It has a very broad application prospect and has a great impact on social progress. In order to ensure the further development of electronic information engineering technology, we should carry out more in-depth research on it, fully understand its role, so that it can provide us with better services.
\end{abstract}

Keywords: Electronic information engineering technology; Application; Future development

\section{1 电子信息工程技术的主要特点}

1.1 智能化

在二十一世纪智能技术是重要的技术之一，所有的新型技术要想得到永续发展都离不开智能技术。现阶段，智能 技术得到了广泛的应用并得到了相应的成果。例如，利用电子信息工程技术实现了对人类思维的拓展，利用计算机来 模仿人类大脑进行思考并形成相应的运行规则, 通过大量数据的计算与学习得到新的能力。从而加快生产效率、提升 生活质量, 并可以对新事物进行进一步开发, 可以对人类的不足进行弥补。

\section{2 网络化}

网络的发展, 手机、电脑已经得到了普及, 可以不用面对面的交流完成各种数据的传送, 以此来突破空间、时间 的束缚, 帮助人们得到更多的信息, 实现信息、数据的共享, 提升数据的传递速度。

1.3 高效化

体能问题是人工劳动中最大的弊端, 在外界因素的影响下会给工作质量带来影响。在充分的利用电子工程信息技 术后可以有效的弥补这一问题。机器只要通过有效的维护可以实现永不停歇, 在使用前做好程序、内容的设定, 可以 有效的避免来自外界的干扰，同时可以提升数据处理规模与处理效率，确保数据储存的准确性。

\section{2 电子信息工程技术的具体应用}

\section{1 日常生活中的应用}

在日常生活中随处可见电子信息工程技术，可以帮助企业抢占市场份额，利用先进的计算技术提升其在市场中的 竞争力, 并对所生产的产品进行有效的创新, 从而提升企业整体效益。从实际生活中不难发现, 现在我们已经无法离 
开电子信息工程技术的辅助, 随着 $5 \mathrm{G}$ 网络的推出, 标志着信息技术已经踏上了新的征程, 可以进一步加快上网速度, 获取数据信息效率更高。比如, 电商平台的启动完全打破了传统消费模式, 使用者可以在电商平台上了解自己所需要 产品的形式、价格等，不但增加了选择面也节省了大量的时间；支付方式的改变，现金交易量变小，使用者可以通过 电子账户完成支付交易, 并可以随时查看所购买的商品与价格, 可以在平台上与商家进行协商, 并对货品进行调换, 增加了交易的便利性。

\section{2 工业生产中的应用}

在全球经济发展的推动下, 我国产业结构也得到了进一步的优化与变革, 工业生产中充分利用了网络化、智能化 与高效化的信息技术, 在此基础上提升了生产效率, 同时也保证了产品的生产质量。以包装行业为例, 若采用人工操 作的方式不仅无法保证工作效率, 也无法保证包装质量, 在一定程度上给企业的经济效益带来直接的影响, 但是使用 纸盒裹包机可以有效的提升工作效率与工作质量, 保证企业的经济效益; 在汽车制造行业中, 由于生产过程中比较复 杂、安装零件较多且结构大小比较难控制, 如果完全依靠人工方式会给拼接工作留下安全隐患, 利用机械自动化技术 可以有效的规避以上问题, 充分利用计算机图像识别技术、机械手臂对安装工作进行有效的控制, 并确保安装的精准 性与工作效率。可以说在工业生产中充分的利用电子信息工程技术可以加快行业转型的速度，并可以提升产品价值。

\section{3 航空航天领域中的应用}

航空航天领域是评定国家军事能力的关键因素, 我国已经成功发射了神舟号与嫦娥号, 并取得了巨大的成就。随 着航空航天领域的不断发展, 我国也逐渐出台了一些了促进航空航天领域发展的高精尖政策, 实现了电子信息工程技 术高质量发展的目标。其中航空航天领域中遥感技术及数据处理技术是核心, 其可以实现对卫星的定位与控制, 并可 以将太空中的图像处理结果、太空物质检测结果进行回传，实现实时共享。

\section{4 工程造价中的应用}

随着建筑行业不断发展, 降电子信息工程技术应用于其中可以实现对不同地区房产图像以及价格进行整合, 实现 消费者远距离参观的目的。同时, 利用计算机技术可以对建筑工程中所使用的材料进行有效的检测、评价, 实现实时 跟踪, 确保其质量与使用安全。此外，由于建筑工程的建筑规模都相对较大，所以使用的资金量、人工、材料等也相 对较多, 在核算过程中若采用人工方式无法确保核算效率与精准性, 而利用电子信息工程技术可以构建起自动化数据 信息管理库，对数据信息进行有效的纠正，并保证其精准性。

\section{5 农业机械中的应用}

随着新农村建设速度不断加快，现代化农村建设也成为国家经济发展中的重点。将电子信息工程技术应用于农业 机械中, 可以有效的提升农业作业效率并可以降低农业生产者的劳动量, 提升农产品的生产质量。利用电子信息工程 技术可以对肥料配比量进行控制, 并根据图像识别构建自动化播撒系统, 有效的控制施肥范围及肥料中各元素的比例。 此外, 在电子信息工程技术的帮助下, 农业生产者不用到现场观察农作物的生长情况, 在计算机远程控制系统的辅助 下来控制肥料比例与农作物的生长情况, 并可以对施肥情况进行优化与调整, 提升农业生产效率。使用现代化农业机 械可以有效的降低农业生产者的生产负担，提升耕作效率，实现农业生产现代化。 ${ }^{[2]}$

\section{3 电子信息工程技术发展过中的问题}

\section{1 国家重视程度不足}

第一, 在现阶段我国电子信息工程技术发展的过程中, 会受到制度、人才方面的影响, 在专业人才培养方面还存 在理念上的偏差，导致电子信息工程技术专业人才缺乏的情况，阻碍了该行业的发展。其次，国家并未给予电子信息 工程类企业发展更加有力的支持，在发展的过程中也没有给予准确的指导，阻碍了企业的发展。

\section{2 缺乏相应的法律法规}

目前, 我国虽然已经制定出相应的法律法规来推动电子信息工程行业的发展, 但是由于观念上的偏差, 导致法律 法规并不完善。侵权情况经常出现在电子信息工程技术研发的过程中, 在法律法规不健全的情况下无法对侵权行为进 行有力的打击, 在一定程度上降低了技术人员的研究积极性, 因此无法在短时间内提升电子信息工程技术的研究水平, 限制了电子信息工程技术的发展与优化。

\section{3 电子信息技术企业发展困难}

由于国家支持力度及重视度相对较低，所以放慢了电子信息工程技术的发展过程，再加之电子信息工程技术在发 展的过程中法律法规的不完善，无法为电子信息工程企业提供稳定的发展环境与有力的社会条件。大多数电子信息工 
程企业在发展中多以自主探索为主, 再加之人才的黄乏, 无法保证企业的整体效益, 在这样一个环境下, 企业人才与 技术都无法得到良好的提升，降低了企业的经济效益。

\section{4 电子信息工程技术未来发展趋势}

4.1 在明确发展目标的基础上, 实现理论的提升

要想确保电子信息工程技术的有效发展, 必须要明确其发展目标, 将理论与实践进行有效的结合。在未来发展的 过程中应明确以下方面。首先, 应充分的将电子信息工程技术与人们的日常生活、工作进行有效的联系, 以此来提升 人们的生活品质与幸福感, 进而促进社会及经济的发展。同时, 可以为人们的生活提供便利, 这也是未来发展的主要 方向。其次，电子信息工程技术与国家发展、经济建设有着紧密的联系，也就是与农业领域、军事领域之间的关系， 在这些领域应用的过程中应更加谨慎。此外, 在电子信息工程技术不断发展的过程中应加大人才培养力度, 确保其与 国家发展要求相符，进一步明确其发展方向，在此基础上对理论进行革新，加快其发展速度。

\section{2 进一步加大创新力度}

构建起良好的技术研发环境。要想为电子信息工程技术创建良好的发展平台并确保其稳步发展, 应强化人才培养, 并加大创新力度。

\section{3 规避安全问题}

电子信息工程技术属于比较重要的技术，但是在使用的过程中会受到一些因素的影响，导致问题出现。主要的问 题为网络信息安全, 当出现这一问题时会导致严重的后果。因此, 在使用此项技术时应重点关注安全问题, 并采取有 效的方式规避安全问题的产生，做好防护工作，建立起防护墙，实现安全使用。

\section{5 结语}

电子信息工程技术可以进一步推动国家的发展与建设，并可以为人们的工作、生活提供更多的便利，改变人们的 工作生活方式。电子信息工程技术更好的实现了网络化、智能化与高效化, 并得到了广泛应用。得到了工业领域、航 空航天领域、工程造价领域等领域的认可与应用, 最大限度的提升了各个领域的经济效益并加快了各个领域的发展速 度, 成为社会、经济发展过程中的重要技术。在未来, 我国要想得到更好的发展, 成为世界强国, 应进一步加大电子 信息工程技术的研发力度，并根据时代发展的要求对其进行创新与优化，加快其发展速度，为国家建设贡献力量。

\section{[参考文献]}

[1]白书铭.浅谈电子信息工程技术的应用与未来发展趋势 [J].科技资讯, 2018 (20): 7-9.

[2]邓志红。电子信息工程技术的应用及未来发展 [J].电子技术与软件工程,2018(20): 251 .

[3] 孔寒冰. 基于本体的工程学科框架研究 [D]. 浙江: 浙江大学, 2009.

作者简介：李云，(1969-）女，研究方向：电子信息。 